\title{
APPLICATION OF MODAL ANALYSIS FOR MECHANICAL DESIGNS OPTIMIZATION
}

\author{
Avadhut Parulekar', S. C. Shilwant ${ }^{2}$

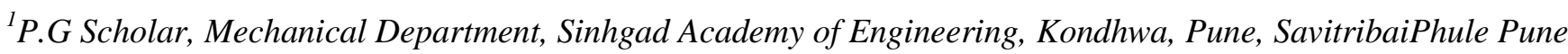 \\ University, Pune \\ ${ }^{2}$ Profesor, Mechanical Department, Sinhgad Academy of Engineering, SavitribaiPhule Pune University, Pune
}

\begin{abstract}
Under dynamic condition, structure components will be excited. If excitation frequency matches with natural frequency of the structure, then resonance takes place. Due to this structure oscillates excessively and may lead to failure. Modal Analysis is one of the major technique to obtain natural frequency of structure and also to obtain different frequency modes. It can be done virtually by using software such as Ansys or Creo. This helps to reduce design time since multiple iterations can be done on design concept without ordering physical components for test. This virtual validation also saves the cost of development since few number of prototypes are required for physical validation.
\end{abstract}

Keywords: Natural frequency, Finite Element Analysis, modal analysis, mode shapes, free-free state modal analysis

\section{INTRODUCTION}

The frequency at which a system tends to oscillate in the absence of any driving or damping force is called Natural Frequency.

$$
f_{n}=\frac{1}{2 \pi} \sqrt{\frac{k}{m}}
$$

Where,

$f_{n}=$ Natural frequency

$k=$ Spring constant

$\mathrm{m}=$ Mass of the system

Modal analysis is an analytical technique that is used to find out natural frequency of the structure. Modal frequency can be defined as the frequency by which structure vibrates after the excitation. Mode shape can be defined as the parameter that represent the deformation of the structure due to the excitation force given by hammer.

Due to increasing automotive pollution, industry is adopting weight reduction techniques. Light weigh components tend to improve fuel economy thus by reducing the amount of harmful emissions left in environment. While weight reduction is focus, it will be important to study changes in dynamic properties of designed structure. It is necessary to check the dynamic behavior of design under different frequency modes. Modal analysis is required to be done for the same to find out natural frequency of the design and frequencies of the component. Hence modal analysis of the component plays important role to decide its dynamic behaviors when vehicle is running. Modal analysis helps us to understand if resonance will take place for modified design and make necessary changes in design to avoid resonance before ordering the test parts.

\section{THEORY}

By Finite Element Analysis (FEA) static structural analysis is first carried out before going for modal analysis. Static structural analysis gives information about stresses at critical areas and displacement values. If design meets the structural analysis then modal analysis is done to understand how design behaves in different frequency ranges and what the modal shapes are.

Input for FEA software is obtained from 3D modeling software such as Creo, Catia, UniGraphics and so on. Preprocessing is done in which 3D geometry is meshed, material properties are assigned and constraints are given. Load is applied as per the functional requirements of the component. Then analysis is run and results are obtained. In post processing, analysis results are reviewed and then acceptability of result is determined depending upon stress values, displacement [1].

Modal analysis is done either in free-free state or constrained state. In free-free state, structure is not constrained. First six modes are ignored as they hardly give any value for frequency. These six modes are three translations around $\mathrm{X}, \mathrm{Y}$ and $\mathrm{Z}$ axis and other three modes are rotations about $\mathrm{X}, \mathrm{Y}$ and $\mathrm{Z}$. So generally seventh mode is considered as natural frequency of structure.

This paper reviews some of the design study for modal analysis of components such as engine mount [1], gear wheel [2], brake disc [3], Impeller for centrifugal pump [4], gear box casting [5], automotive chassis [6]. 
SahilNaghate and Sandeep Patil [1] analyzed engine mount to find its natural frequency and then to study its geometry for two different materials. One is Aluminum Alloy while as other is Magnesium Alloy. Engine has most wide range of frequency pattern due to rotating components at low to high RPM. Natural frequency of engine mount needs to be different than any of these frequencies to avoid resonance. Structural analysis is done and found that von-mises stresses $(26.758 \mathrm{MPa})$ are below yield limits of material. Deflection $1.6258 \mathrm{~mm}$ is also within acceptable limits. Modal analysis is done by constraining the bracket in a way it will be mounted during engine assembly. Four modes of vibrations are considered. Frequencies found are respectively $147.78 \mathrm{~Hz}$, 213.26 Hz, $322.78 \mathrm{~Hz}$ and $630.5 \mathrm{~Hz}$.

All the analysis is again conducted on Magnesium Alloy engine mount bracket and then results are compared with Aluminum Alloy.

It is observed that stresses are almost same for both the brackets but deformations are varying. Magnesium alloy bracket has higher deflection since its density is lesser then Aluminum Alloy [1].

Andrea Cizikoval and Katarina Monkova1 from Technical University of Kosice conducted virtual and experimental modal analysis on gear wheel that is used in planetary gear system [2]. Piezoelectric accelerometer was used to measure the vibrations. Modal hammer was used to induce the vibrations in gear wheel. Excitation point was identified on gearwheel by creation of geometry in MTC-hammer software. First eleven mode shapes of gear wheels were compared with experimental analysis.

Brake Disc of motor cycle is analyzed for natural frequency. Due to frictional forces, brake disc vibrations are generated in disc and squealing takes place. Squealing is noise generation while applying the brakes. Total four different disc geometries were analyzed for its natural frequencies and deformations at different modes. It is observed that as weight of the component decreases its natural frequency increases, for same material[3].

MaratheShalin , Patel Vishal, Patel Harshkumar, Patel Dhruvinkumar studied modal analysis for the backward bladed impeller of centrifugal pump [4]. Blades of the centrifugal are subjected to high fatigue failure and if any one blade fails at high RPMs then it may cause a major damage to the pump and surrounding components. Experimental testing is done to find out if different magnitude forces affect the natural frequency of the structure. It was found natural frequency was unaltered for different magnitude forces. Numerical modal analysis was done by using Creo 2.0 software where elements used for meshing were tetrahedral and element size was 10.
Maximum deformation found from 10 modes was $1 \mathrm{~mm}$ and was acceptable [4].

Three Stage Industrial Helical Gearbox casing [5] was analyzed for its natural frequency and mode shapes. It consists of top and bottom housing and catcher cover. As this case supports gears, shafts and bearings that are used to transmit high torque, it is utmost important to analyze this case for its natural frequency and dynamic behavior. Material used for casing is grey forged iron FG260 (for top and bottom housing) and FG220 (catcher cover).Operating frequency of gears is compared with natural frequency of casing. Natural frequency is obtained by using 'Free-Free' conditions. In this analysis, no constraints are applied to the design and natural frequency is found out. As first six modes show zero frequency, additional four modes were considered to find out frequency and mode shapes [5].

Chassis is one of the most important structural component of any automotive. It experiences variety of forces acting on it and different frequency patterns due to road irregularities and variety of engine loading conditions. It is very important to analyze dynamic behaviors of chassis under different loading conditions and frequency patters to avoid any failure in chassis that may lead to an accident and ensure safety of the occupants.

Dr.R.Rajappan, and M.Vivekanandhan [6] did modal analysis study for truck chassis in which they analyzed dynamic mode shapes. 4 node Shell element were used for meshing and first 6 frequencies were considered. Also study was done for different engine speeds and natural frequencies. Free-free state modal analysis is done. First 30 frequency modes are considered for result. It has been found that chassis experiences torsional, lateral and vertical bending [6].

\section{FUTURE SCOPE}

This review study was done to gain basic knowledge about virtual modal analysis and process to do it using FEA software. Vibrations in automotive vehicles are generated via reactions between road and tyre contact. Engine is another resource that will generate the vibrations. If frequency generated due to these vibrations matches with natural frequency of any of the components, then resonance takes place. This leads to unwanted noise between structural components due to metal to metal contact. This is major cause of customer discomfort. If such resonance takes place between moving components such as shaft, then it may lead to serious system failure leading to severe accident of the vehicle.

I will be developing new concept for fire extinguisher bracket. This bracket will be used on combine which is an agriculture product. Combine has enormous 13.0litre engine that generates variety of frequencies. 


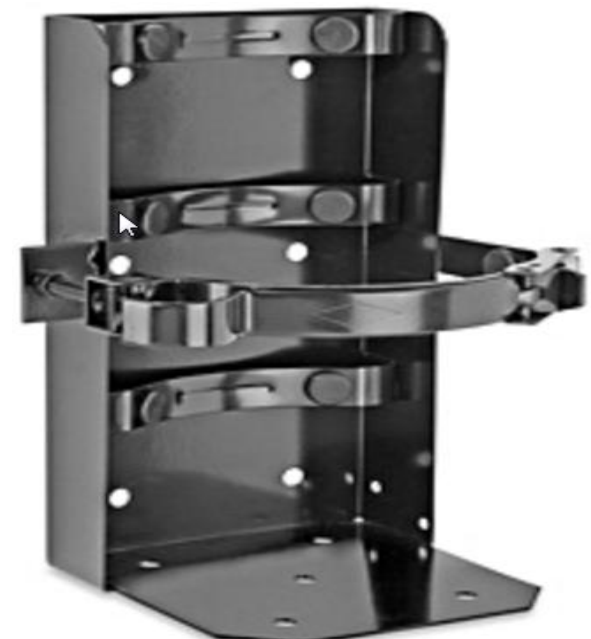

Fig 1: Current Fire Extinguisher Bracket

This leads to study of natural frequency for newly designed components and perform the modal analysis. It will give safe design.

Virtual modal analysis using advanced simulation software such as Creo, Ansys etc will help to save the design iteration time. It is planned to perform the analysis on various design concepts and choose the best option. It is really not required to do physical validation of all the concepts to evaluate their frequency analysis. This greatly saves time, efforts and overall development time will get reduced. We will be analyzing different concepts of fire extinguisher bracket and will evaluate their natural frequencies.

Analysis will be done by both free-free and constrained methods to study difference in frequency values obtained. Analysis will be performed mainly for sheet metal and casting concepts. It will also be planned to study how the natural frequency varies with different materials and if density of material has any effect on its natural frequency and modal properties. Creo 2.0 software will be used to model the 3D concepts and then do the simulation. Current design for fire extinguisher bracket will be evaluated as well for its modal properties and natural frequency. This will provide good 1:1 comparison between current design and proposed concepts. Attempt will be made to keep mounting locations for proposed design same as current design. It will eliminate the requirement to modify other mounting assemblies and components for this bracket.

One more peculiarity of new design is that, it will accommodate variety of fire extinguishers on same bracket. Diameter for fire extinguishers vary from $125 \mathrm{~mm}$ to 185 $\mathrm{mm}$ and height varies from $450 \mathrm{~mm}$ to $620 \mathrm{~mm}$. Heaviest fire extinguisher is of weight $20 \mathrm{~kg}$. So worst load case for the bracket will be $20 \mathrm{~kg}$ fire extinguisher with $620 \mathrm{~mm}$ diameter. For static structural analysis three load cases will be considered. Those are 3G vertical load, lateral load and fore aft load. Generally G load is considered as gravity weight exerted by fire extinguisher cylinder on fire extinguisher bracket. To simulate dynamic conditions, where there will be more load due to dynamic conditions more loading is considered that is generally 3 times the G load and is called as $3 \mathrm{G}$ loading.

Once concept is finalized, prototype parts will be ordered and they will be tested in lab as well on durability build machines. Life expectancy for this bracket is same as vehicle life which is 10 years. Here vehicle considered is Combine. Fire extinguisher to be held in position by using two nylon straps. For strap, ultra violet stability is important criterion since fire extinguisher bracket will be exposed to extreme heat during summer. As well in some cases there will be heat passed from engine to the belt. So durability of the belt and its tension holding capacity is important to evaluate. At no point, belt should lose its tension. That leads to the risk of fire extinguisher fall down. That's why belt will be tested in lab on proposed fire extinguisher bracket concept. These nylon straps will be off the shelf option from supplier and hence won't be evaluated in analysis. Strap will consists of steel buckles and will be used to tighten the belt around fire extinguisher bracket. Shaker table test will be carried out in test lab if bracket is not tested in field. Once design is validated, then it will be implemented on current combine product.

\subsection{Proposed Experimental Setup}

It will consist of test fixture to hold the fire extinguisher bracket in position. Then by using actuator, 5G load will be applied on bracket in regular intervals. This process will be repeated for 500 cycles. During this process bracket should not deform permanently and no cracks should be seen on it.

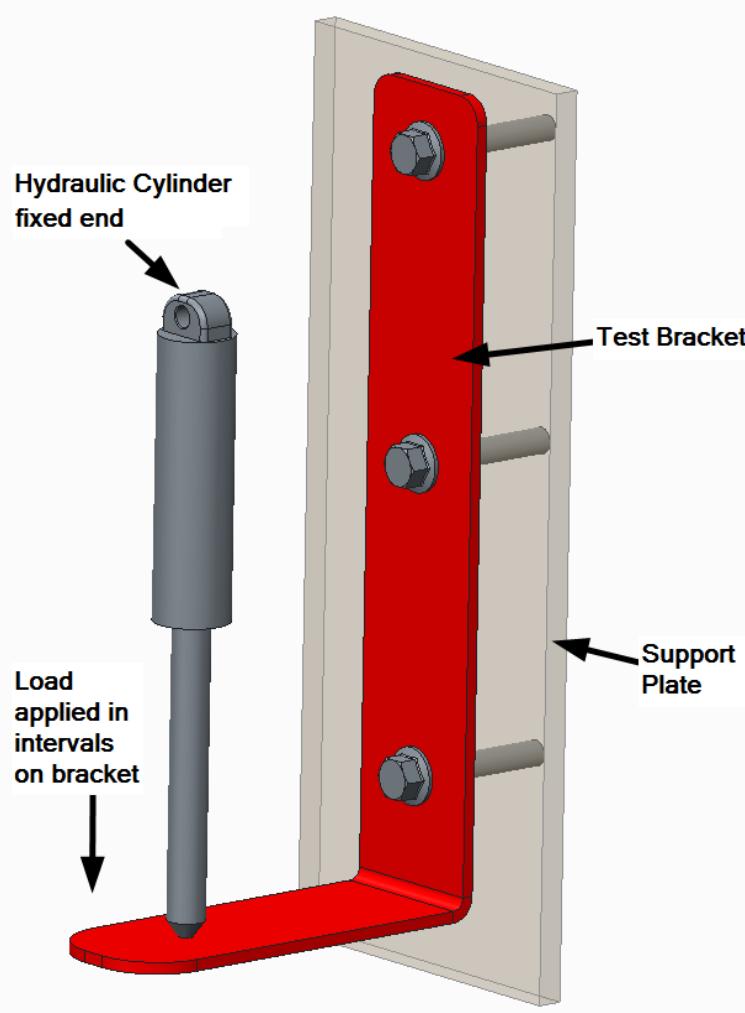

Fig 2: Experimental test Setup 
After 500 cycles are done, then load will be gradually increased to $6 \mathrm{G}, 7 \mathrm{G}$ and so on. Finally bracket will deform permanently and will crack at certain load. This will provide maximum acceptable loading criteria for bracket.

This maximum loading criteria will be helpful to install the fire extinguisher on vehicle. If any specific structural area of vehicle is seeing the $\mathrm{G}$ load at which bracket failed during testing, then that area can be avoided and some other area on vehicle where less load, will be used to mount the bracket.

Instead of building separate test stand in the lab, this bracket will be tested in standard laboratories in USA or Europe. These labs are specialized in testing the fire extinguishers and its mountings. They have readymade set ups available to mount variety of brackets. Depending upon parts availability from supplier test lab schedule will be decided.

\section{CONCLUSION}

Modal Analysis is one of the important analysis as it determines dynamic behavior of the design under different loading and frequency conditions. It helps to understand what natural frequency of the designed structure is and modify it to avoid resonance with surrounding components. It is great method to reduce new design development time and allows to do many iterations to refine the design in early stages. This helps in reducing in overall design time and saves cost as well.

\section{REFERENCES}

[1]. SahilNaghate, Sandeep Patil "Modal Analysis of Engine Mounting Bracket Using FEA,'IEEE International Journal of Engineering Research and Applications (IJERA) ISSN: 2248-9622 www.ijera.com Vol. 2, Issue4, July-August 2012, pp.1973-1979

[2]. Andrea Cizikova1, Katarina Monkova1, "Numerical And Experimental Modal Analysis Of Gear Wheel," DOI: 10.17973/MMSJ.2016_11_201654 pp 1232-1236

[3]. Mr. Pravin N. Jawarikar, Dr. Subim N. Khan, Mr. Balaji

D. Kshirsagar, "Structural optimization, thermal and Vibration analysis of two wheeler disc Brake rotor," in ICRIESHM ISBN:978-93-86171-02-3 Page 336-353

[4]. MaratheShalin , Patel Vishal, Patel Harshkumar, Patel Dhruvinkumar, "Experimental and numerical modal analysis of The backward bladed impeller of centrifugal Pump," in IJARESM ISSN : 2394-1766

[5]. Balasaheb SahebraoVikhe, "Design Analysis Of Industrial Gear Box Casing," in International Research Journal of Engineering and Technology (IRJET) Volume: 03 Issue: 11 | Oct -2016 p-ISSN: 2395-0072

[6]. Dr.R.Rajappan, M.Vivekanandhan, ,"Static and Modal Analysis of Chassis by Using Fea," in International Research Journal of Engineering and Technology (IRJET) Volume: 03 Issue: 11 | Oct -2016 p-ISSN: 2395-0072The International Journal Of Engineering And Science (Ijes)||Volume || 2 ||Issue|| 2 ||Pages|| 63-73 ||2013||Issn: 2319

- 1813 Isbn: 2319 - 1805 